\title{
Study on the Feasibility and the Safety of Hem-o-lok Clipping for Complicated Acute Appendicitis during Laparoscopic Appendectomy
}

\author{
Ki Hyun Kim, M.D., Si Hak Lee, M.D., Kwang Ho Yang, M.D., Sun Hwi Hwang, M.D. \\ Department of Surgery, Pusan National University Yangsan Hospital, Yangsan, Korea
}

Purpose: The objective of this retrospective study is to evaluate the practical feasibility and safety of applying hem-o-lok clips for the closure of the appendiceal stump in complicated appendicitis.

Methods: From January 2009 to April 2010, 267 patients who underwent laparoscopic appendectomies at the Pusan National University Yangsan Hospital were included in this study. Of these patients, 161 were diagnosed by computed tomography as having complicated acute appendicitis. In 107 patients, the appendiceal stump was closed with hem-o-lok clips, whereas in the remaining 160 patients, it was closed with endoloops. These two groups were compared for clinicopathologic data and procedural cost-effectiveness.
Results: No significant differences were found between the two groups in terms of age, sex, hospital stay, or blood loss. Furthermore, postoperative complications were similar and highly acceptable in both groups. However, operation times were significantly shorter and hospital cost was lower for the hem-o-lok group.

Conclusion: The hem-o-lok clips has advantages such as simplicity of application, a shorter operation time, and lower hospital cost when used as a means of securing the base of the appendix in relation to the endoloop procedure.

Key words: Laparoscopic appendectomy, Hem-o-lok clip, ENDOLOOP

\section{INTRODUCTION}

Appendicitis is the most common cause of acute abdomen, and appendectomy is associated with significant morbidity. ${ }^{1}$ Laparoscopic appendectomy has been accepted as a standard treatment modality and its usage is increasing, although a comparatively recent analysis of 259,735 appendectomies performed in British NHS hospitals from 1996 to 2006 showed that laparoscopic appendectomy accounted for only $6.3 \%$ of all procedures performed. ${ }^{2}$ Nevertheless, laparoscopic appendectomy has been shown to be safe, and is associated with fewer postoperative complications and faster recovery than open appendectomy. ${ }^{3}$ However, the risk of intra-abdominal abscess development after laparoscopic appendectomy is still a matter of debate, as comparative studies on laparoscopic and open appendectomy have produced variable results, that is, from no difference to an increase after laparoscopic appendectomy. ${ }^{4,5}$ Beldi et al. ${ }^{6}$ described a significantly higher rate for intra-abdominal

Received January 7, 2013, Revised March 6, 2013,

Accepted March 13, 2013

※ Corresponding author : Sun Hwi Hwang

Department of Surgery, Pusan National University Yangsan

Hospital, Kumoh-ro, Mulgum-up, Yangsan 626-770, Korea

Tel : +82-55-360-2124, Fax : +82-55-360-2473

E-mail : hwangsh@pusan.ac.kr

http://dx.doi.org/10.7602/jmis.2013.16.1.6 surgical site infections in their ENDOLOOP group in a non-concurrent cohort study.

The technical focus in laparoscopic appendectomy is largely on the treatment of appendiceal stump closure. The majority of surgeons use a stapler ${ }^{7}$ or a loop ${ }^{8}$ to close the appendiceal stump, but although the stapler is safe and fast, it is expensive. On the other hand, the loop is cheaper but it requires a higher level of laparoscopic training. In addition, lost stapler clips have been shown to cause peritoneal adhesions and have been associated with small-bowel obstruction, and hence, ileus. ${ }^{9}$

Open appendectomy was the standard treatment for appendicitis for more than a century, but since the advent of minimal invasive surgery during the late 1980s, laparoscopic appendectomy has been adopted by many surgeons and institutions as a means for removing the inflamed appendix. ${ }^{1,3}$ Acute appendicitis is one of the most common emergent diseases that surgeons encounter in daily practice. However, complicated appendicitis can result in significant morbidity. ${ }^{4-6}$ Complicated appendicitis with a perforation, an abscess, or generalized peritonitis is still associated with increased postoperative morbidity, whereas uncomplicated appendicitis has a low complication rate. $^{10}$

Control of the appendiceal stump is crucial during laparoscopic appendectomy, and is commonly achieved using ENDOLOOPs or endovascular gastrointestinal anastomosis (GIA) stapling device. The main objections on the laparoscopic approach 
are higher hospital costs, due to the use of disposable instruments, longer operations, and a higher rate of intra-abdominal abscess formation, in particularly in cases of perforated appendicitis. ${ }^{11}$ However, a meta-analysis of randomized controlled trials that compared laparoscopic and open appendectomy suggested benefits for laparoscopic surgery. ${ }^{12,13}$ Many different technics to close the appendiceal stump closure. ${ }^{14-16}$ However, no meta-analysis has been performed on the outcomes of appendectomy performed using Hem-o-lok clips or ENDOLOOPs to secure the stump of the appendix.

Given this background, we describe our experiences of these clips for appendiceal stump closure, and the results of our analysis of the merits of Hem-o-lok clips for appendiceal stump closure during laparoscopic appendectomy in complicated appendicitis.

\section{MATERIALS AND METHODS}

From January 2009 to April 2010, 267 patients with clinically suspected acute appendicitis underwent laparoscopic appendectomy at our institution. Three of the seven surgeons involved used Hem-o-lok clips (Non-absorbable Polymer Ligating Clips, Teleflex Medical, RTP, NC, USA) whenever possible, whereas the other four surgeons preferred the ENDOLOOPs (Ligature made with Coated VICRYL, ETHICON, INC.). Both groups of surgeons had the same level of expertise of laparoscopic appendectomy.

In order to stratify the severity of inflammation, all cases were also classified, based on computed tomography findings and we defined complicated acute appendicitis as more than grade 3 (Table 1). ${ }^{2}$ Complicated acute appendicitis was defined based on intraoperative status, that is, acute inflammation of the appendix with a reddish aspect of surrounding peritoneum (localized peritonitis), a perforation of the appendix, the presence of pus or of a fibrin membrane around the appendix, or the presence of a frank periappendiceal abscess.

Surgical procedures were conducted as previously described. ${ }^{17}$ Briefly, the proximal base of the appendix was closed using $10 \mathrm{~mm}$ double Hem-o-lok clips or ENDOLOOPs (Fig. $1)$.

The largest appendicular stump diameter that can be safely closed with a Hem-o-lok clip is $12 \mathrm{~mm}$. When difficultly was experienced confirming a perforated appendicitis or to deliver more distal portion of the appendix although the base of the appendix is accessible, retrograde appendectomy was performed in the Hem-o-lok group. ${ }^{18}$ This consisted of dividing the appendiceal base between clips, and then dividing the mesoappendix until the appendiceal tip was reached.

All relevant patient data, which included details of complications during hospitalization, histology reports, and final diagnoses, were stored in a database.

\section{RESULTS}

Appendectomy was performed in 335 patients between January 2009 and April 2010 at our institution. Table 2 shows the characteristics of the patients that underwent laparoscopic appendectomy, which was performed in 267 (79.7\%) patients with appendicitis and open appendectomy was performed in the remainder. All the operations were performed by the operator's preference which are laparoscopic or open surgical techniqs. There was no conversion to open surgery. Laparoscopic appendectomy with stump closure using Hem-o-lok clips was performed in 107 (40.1\%) patients, ENDOLOOPs was used in the other 160 (59.9\%). Our analysis of patient data revealed no significant differences between the Hem-o-lok clip group and the ENDOLOOP group with the exception of hospital cost and operation time. The mean hospital cost in the Hem-o-lok group was $806.7 \pm 178.7$ dollars, whereas in the ENDOLOOP group it was $972.7 \pm 355.8$ dollars, and this difference was significant

Table 1. CT grades for appendicitis

\begin{tabular}{|c|c|c|}
\hline Grade & CT definition & CT findings \\
\hline 0 & Normal & $\begin{array}{l}\text { Fluid-filled appendix smaller than } 6 \mathrm{~mm} \text { or gas-filled appendix smaller than } 8 \mathrm{~mm} \\
\text { with thin wall }\end{array}$ \\
\hline 1 & Probable appendicitis & Fluid-filled appendix $6 \sim 7.9 \mathrm{~mm}$ without periappendiceal abnormality \\
\hline 2 & Appendicitis & $\begin{array}{l}\text { Fluid-filled appendix } 6 \mathrm{~mm} \text { or larger with enhancing wall but without periappendiceal } \\
\text { stranding }\end{array}$ \\
\hline 3 & Appendicitis with periappendicitis & Fluid-filled appendix $6 \mathrm{~mm}$ or larger and periappendiceal stranding \\
\hline 4 & Appendicitis with rupture & Fluid-filled appendix $6 \mathrm{~mm}$ or larger and periappendiceal fluid \\
\hline 5 & Complicated appendicitis & Abscess or inflammatory mass \\
\hline
\end{tabular}



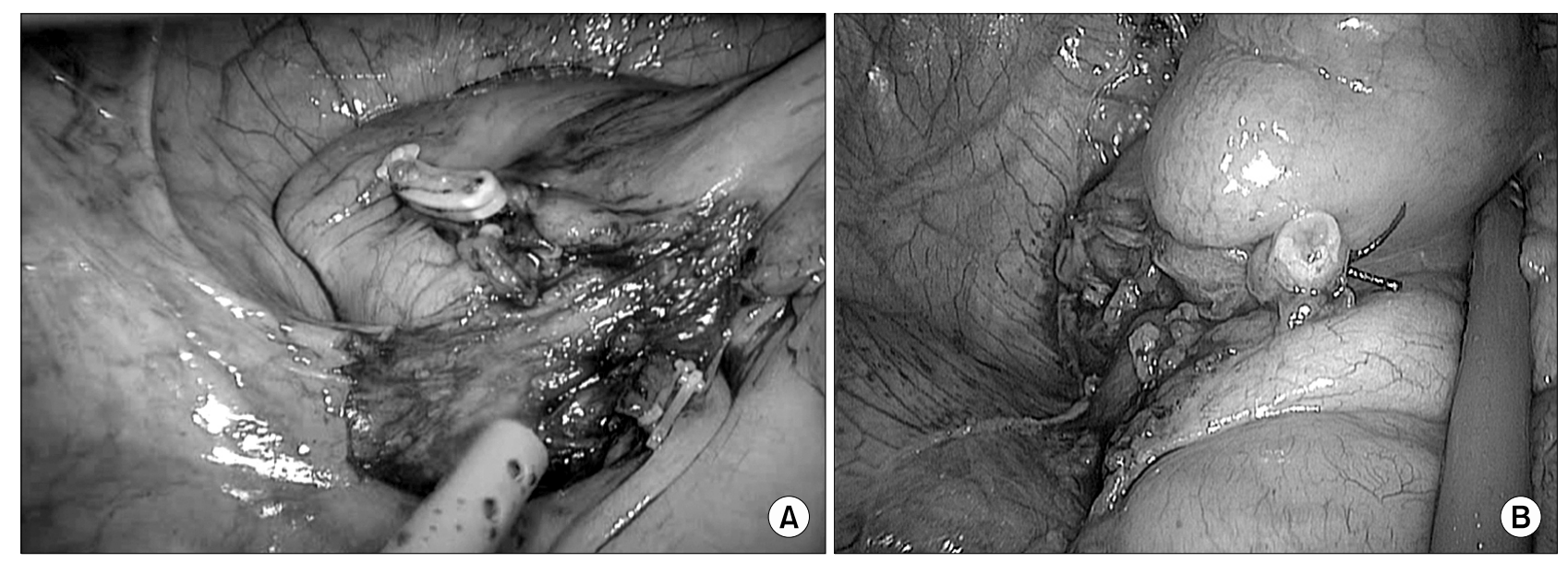

Fig. 1. (A) Hem-o-lok clip. (B) ENDOLOOP.

Table 2. Clinicopathologic characteristics of patients who underwent laparoscopic appendectomy

\begin{tabular}{|c|c|c|c|}
\hline Characteristics & Hem-o-lok (n=107) & ENDOLOOP $(n=160)$ & $p$ value \\
\hline Conversion to open surgery & 0 & 0 & \\
\hline Successful laparoscopic surgery & 107 & 160 & \\
\hline $\operatorname{Sex}(M: F)$ & $54: 53$ & $78: 82$ & 0.73 \\
\hline Mean age (years) & $31.1 \pm 20.4$ & $30.09 \pm 20.0$ & 0.75 \\
\hline Body mass index $\left(\mathrm{kg} / \mathrm{m}^{2}\right)$ & $21.7 \pm 3.3$ & $23.1 \pm 4.0$ & 0.23 \\
\hline OP time (min) & $47.3 \pm 23.1$ & $76.63 \pm 25.1$ & 0.04 \\
\hline Hospital stay & $4.1 \pm 1.8$ & $4.2 \pm 2.4$ & 0.68 \\
\hline Cost (dollars) & $806.7 \pm 178.7$ & $972.7 \pm 355.8$ & 0.04 \\
\hline Cost (won, 1 dollar=1,086 won) & $876,076.2 \pm 194,068.2$ & $105,635.2 \pm 386,398.8$ & 0.04 \\
\hline Blood loss & & & 0.78 \\
\hline$<100 \mathrm{ml}$ & 96 & 145 & \\
\hline$\geq 100 \mathrm{ml}$ & 11 & 15 & \\
\hline Appendicitis on $\mathrm{CT}$ exam & & & 0.11 \\
\hline Normal (Grade 0) & 1 & 6 & \\
\hline Probable appendicitis (Grade 1) & 2 & 14 & \\
\hline Appendicitis (Grade 2) & 36 & 47 & \\
\hline Appendicitis and periappendicitis (Grade 3) & 28 & 34 & \\
\hline Appendicitis with rupture (Grade 4) & 30 & 44 & \\
\hline Complicated appendicitis (Grade 5) & 10 & 15 & \\
\hline
\end{tabular}

$(p=0.04)$. The average operative time was $47.3 \mathrm{~min}$ in the Hem-o-lok group and $76.6 \mathrm{~min}$ in the ENDOLOOP group, and these were also significantly different $(p=0.04)$. No significant inter-group differences were found with respect to grade of appendicitis by preoperative $\mathrm{CT}$ and postoperative findings.

The perioperative outcomes of complicated acute appendicitis cases are listed in Table 3. No significant differences were observed between the two study groups with respect to post-operative outcomes. However, average operative times were 43.5 and 94.5 minutes in the Hem-o-lok and ENDOLOOP groups.
Postoperative morbidities are listed in Table 4; no mortality occurred in either group. Furthermore, postoperative surgical complication rates were similar in the two groups; complications occurred in 3 patients in the Hem-o-lok group (2.7\%) and in 5 patients in the ENDOLOOP group (3.1\%). Bleeding from the mesoappendiceal artery was treated by reoperation. For all others complications were treated nonoperatively.

\section{DISCUSSION}

The use of clips to close the appendiceal stump was first 
Table 3. Comparison between hem-o-lok clipping and ENDOLOOP ligation in complicated appendicitis

\begin{tabular}{lccc}
\hline & $\begin{array}{c}\text { Hem-o-lok } \\
(\mathrm{n}=68)\end{array}$ & $\begin{array}{c}\text { ENDOLOOP } \\
(\mathrm{n}=93)\end{array}$ & $p$ value \\
\hline OP time (min) & $43.5 \pm 16.7$ & $94.5 \pm 56.6$ & 0.03 \\
Hospital stay (d) & $4.4 \pm 1.2$ & $6.5 \pm 5.1$ & 0.07 \\
Resumption to diet (d) & $2.1 \pm 1.5$ & $3.1 \pm 1.7$ & 0.07 \\
Antibiotics used (d) & $3.1 \pm 2.1$ & $3.5 \pm 2.1$ & 0.06 \\
Reoperation & 1 & 0 & $\mathrm{NA}$ \\
\hline
\end{tabular}

described by Cristalli et al. 1991 who used metal clips, ${ }^{15}$ but this technique did not achieve general acceptance. More recently, the use of simple non-absorbable clips for appendicular stump closure (Hem-o-lok MLX polymeric clips, Weck Closure Systems, NC, USA) was suggested by Hanssen et al. ${ }^{16}$ and Delibegovic and Matovic. ${ }^{19}$ This approach simplifies the procedure and resembles the cystic duct clipping performed during laparoscopic cholecystectomy. Furthermore, hospital costs for cholecystectomy and Hem-o-lok clips are almost identical.

Many studies have demonstrated significantly higher hospital costs for laparoscopic appendectomy than open appendectomy, ${ }^{20,21}$ which is mainly due to the use of staplers and specimen bags. ${ }^{17}$ In the present study, total cost of simple laparoscopic appendectomy was found to be reduced by $806.7 \pm 178.7$ dollars when a clip was used instead of an ENDOLOOP without increasing the complication rate. Hem-o-lok clip is not only cheaper than ENDOLOOP but also easier than ENDOLOOP. Furthermore, operating time seemed to be shorter Hem-o-lok clip group than ENDOLOOP group. There are comparative study of Hem-o-lok clips and staples. ${ }^{16}$ Hem-o-lok clip group. Also Hem-o-lok clips are cost-effective surgical procedure. However, surgery was longer than staples group.

We perform retrograde appendectomy when the appendix is adherent around the cecum. Its advantages include its simplicity and speed. Retrograde appendectomy avoids the risk of rupture and of soiling the operative field. We believe that retrograde appendectomy and the use of a stepwise approach to laparoscopic appendectomy, as described previously, markedly facilitates the removal of a difficult appendix. ${ }^{7}$ It should be noted that we create only a small mesenteric window next to the base of the appendix, using only minimal blunt dissection and an endoscopic right angle. When used appropriately, we believe that this technique helps prevent some of the infective and mechanical complications that occur after "difficult" appendectomy.
Table 4. Postoperative morbidity after laparoscopic appendectomy in complicated acute appendicitis

\begin{tabular}{lcc}
\hline \multicolumn{1}{c}{ Variable } & Hem-o-lok & Endo-loop \\
\hline Intra-abdominal abscess & 0 & 2 \\
Fever without fluid collection & 0 & 1 \\
Surgical site infection & 1 & 0 \\
Port site hernia & 0 & 0 \\
Prolonged antibiotics treatment & 1 & 2 \\
Postoperative bleeding & 1 & 0 \\
\hline
\end{tabular}

\section{CONCLUSION}

This study shows that the treatment of the appendiceal base can be performed safely and cost-effectively using a Hem-o-lok clip in acute appendicitis or complicated appendicitis patients. Therefore we recommend the use of a Hem-o-lok clip for the closure of the appendicular stump as the good procedure in laparoscopic appendectomy.

\section{REFERENCES}

1) Simpson J, Samaraweera AP, Sara RK, Lobo DN. Acute appendicitis--a benign disease? Ann R Coll Surg Engl 2008; 90:313-316.

2) Faiz O, Clark J, Brown T, et al. Traditional and laparoscopic appendectomy in adults: outcomes in English NHS hospitals between 1996 and 2006. Ann Surg 2008;248:800-806.

3) Pedersen AG, Petersen OB, Wara P, Ronning H, Qvist N, Laurberg S. Randomized clinical trial of laparoscopic versus open appendectomy. Br J Surg 2001;88:200-205.

4) Piskun G, Kozik D, Rajpal S, Shaftan G, Fogler R. Comparison of laparoscopic, open, and converted appendectomy for perforated appendicitis. Surg Endosc 2001;15:660-662.

5) Kouwenhoven EA, Repelaer van Driel OJ, van Erp WF. Fear for the intraabdominal abscess after laparoscopic appendectomy: not realistic. Surg Endosc 2005;19:923-926.

6) Beldi G, Vorburger SA, Bruegger LE, Kocher T, Inderbitzin $\mathrm{D}$, Candinas D. Analysis of stapling versus endoloops in appendiceal stump closure. Br J Surg 2006;93:1390-1393.

7) Raptopoulos V, Katsou G, Rosen MP, Siewert B, Goldberg SN, Kruskal JB. Acute appendicitis: effect of increased use of CT on selecting patients earlier. Radiology 2003;226:521526.

8) Partecke LI, von Bernstorff W, Karrasch A, et al. Unexpected findings on laparoscopy for suspected acute appendicitis: a pro for laparoscopic appendectomy as the standard procedure for acute appendicitis. Langenbecks Arch Surg 2009;23:28512854. 
9) Losanoff JE, Kjossev KT. A new technique for retrograde appendectomy. Eur J Surg 1999;165:268-269.

10) Wagner M, Aronsky D, Tschudi J, Metzger A, Klaiber C. Laparoscopic stapler appendectomy. A prospective study of 267 consecutive cases. Surg Endosc 1996;10:895-899.

11) Arcovedo R, Barrera H, Reyes HS. Securing the appendiceal stump with the Gea extracorporeal sliding knot during laparoscopic appendectomy is safe and economical. Surg Endosc 2007;21:1764-1767.

12) Kuehnel F, Marusch F, Koch A, Gastinger I. Retained loose linear cutter staples after laparoscopic appendectomy as the cause of mechanical small bowel obstruction. Int J Colorectal Dis 2007;22:717-718.

13) Garg CP, Vaidya BB, Chengalath MM. Efficacy of laparoscopy in complicated appendicitis. Int J Surg 2009;7:250-252.

14) Aziz O, Athanasiou T, Tekkis PP, et al. Laparoscopic versus open appendectomy in children: a meta-analysis. Ann Surg 2006;243:17-27.

15) Cristalli BG, Izard V, Jacob D, Levardon M. Laparoscopic appendectomy using a clip applier. Surg Endosc 1991;5:176-
178.

16) Hanssen A, Plotnikov S, Dubois R. Laparoscopic appendectomy using a polymeric clip to close the appendicular stump. JSLS 2007;11:59-62.

17) Paik PS, Towson JA, Anthone GJ, Ortega AE, Simons AJ, Beart RW Jr. Intra-abdominal abscesses following laparoscopic and open appendectomies. J Gastrointest Surg 1997;1:188192.

18) Slim K, Pezet D, Chipponi J. Laparoscopic or open appendectomy? Critical review of randomized, controlled trials. Dis Colon Rectum 1998;41:398-403.

19) Delibegovic S, Matovic E. Hem-o-lok plastic clips in securing of the base of the appendix during laparoscopic appendectomy. Surg Endosc 2009;23:2851-2854.

20) Cueto J, D'Allemagne B, Vazquez-Frias JA, et al. Morbidity of laparoscopic surgery for complicated appendicitis: an international study. Surg Endosc 2006;20:717-720.

21) Binnebosel M, Otto J, Stumpf M, et al. Acute appendicitis. Modern diagnostics--surgical ultrasound. Chirurg 2009;80:579587. 